\title{
Pirsgirêkên Beşa Ziman û Edebiyata Kurdî di Zanîngehên Tirkiyeyê de*
}

The Problems of Kurdish Language and Literature Departments in Turkey

\section{Nesim SÖNMEZ ${ }^{1}$}

Received: January 4, 2015 Accepted: January 26, 2015

\begin{abstract}
In Turkey, for the first time, on December 10, 2009, the Institute of Living languages in Mardin Artuklu University was established and the education of Kurdish Language was commenced. After the establishment of this institute, in some of the cities of the region such as Muş, Van and Bingöl, institutes were established. The establishment of these education organizations was of great significance for Kurdish people, consequently, these establishments gave people of the region and Turkey great excitement. When the announcement for master education was proclaimed, hundreds of people, with immense exhilaration applied for the exams to be held. Some of them succeeded in the exams and started to have their education. The Education Minister and the chairman of the council of higher education announced that "...those finishing the program would have right to be the teacher of Kurdish Language". Hundreds of people have finished this program but unfortunately just 28 of them became teachers. Furthermore, in order to obtain academic to teach at universities, there is a great necessity for staff. It is also a huge problem waiting to be solved. In some of universities, the Kurdish language and literature departments were started under the umbrella of Faculty of Literature (Letters) and some are waiting to be established. There are many obstacles in this aspect to be solved, as well. Some departments for the Culture and Literature Kurdish were established, however, there are many problems in terms of place, library, study saloons and conference halls, education materials and etc., it needs to be noticed and be solved in the shortest time available. Our objective in this article is to focus on these problems and to offer some solutions. If the existing problems culture and language mentioned are solved, we strongly believe that god opportunities will emerge and it will give rise to the peace and brotherhood process for the peoples.
\end{abstract}

Keywords: institute, Literature, Department, Kurdish, Language, Living

Recommended citation:

Sonmez, N. (2016). Pırsgırêkên beşa zıman û edebıyata Kurdî dı zanîngehên Tırkıyeyê de [The Problems of Kurdish Language and Literature Departments in Turkey]. International Journal of Kurdish Studies 2 (1), pp. $96-104$.

\footnotetext{
* Ev gotar hanê cara yekem di 11-12 Hezîran 2015an de li Zanîngeha Bîngolê di sempozyuma "Toplumsal Barışın Sağlanması ve Sonrası" de hatiye pêşkeşkirin. Me ev gotar cara duduyan di ber çavan re derbaskir û berfirehtir kir paşê jî di vî kovarê de da weşandin.

${ }^{1}$ Asst. Prof. Dr., Vice Director of Institute of Living Languages and The Chairman of Kurdish language and Culture Dpt., Yuzuncu Yil University, Van, Turkey.
} 


\section{Kurte}

Li Tirkiyeyê cara yekemin, di 10'ê meha Berfanbarê ya sala 2009'an de li Mêrdînê, di Zanîngeha Artukluyê de bi navê Enstîtuya Zimanên Zindî enstîtuyek hat avakirin û li vê enstîtuyê xebatên perwerdehiya zimanê kurdî dest pêkir. Li dû vê enstîtuyê, li hinek bajarên herêmê wek Mûş, Wan û Bîngolê jî enstîtu hatin vekirin. Avakirina van saziyên perwerdehiyê, ji bo Gelê Kurd tiştekî gelek girîng bû lewra van avakirinan kelecanek zêde zêde da hemwelatiyên herêmê û yên Tirkiyê. Dema ku ji bo serlêdanên xwendekarên lîsansa bilind îlan hatin dayîn bi sedan kes, bi kelecanek gewre, serî li azmûnan dan. Hinek ji wan di van azmûnan de bi ser ketin û dest bi perwerde ya xwe kirin. Wezîrê perwerdehiyê û Serokê Lijneya Hindekariya Bilind di serî de ji bo vê bernameyê digot ku: "Ên vê bernameyê xilas bikin dikarin bibin mamosteyê zimanê kurdî". Bi sedan kesî ev bername xilas kirin lê mixabin ji wan tenê 28 kes bûne mamoste. Dîsa ji bo peydakirina mamosteyên ku di zanîngehan de dersan bidin, hewcedariyek mezin bi qadroyan hene. Ev jî pirsgirêkek gelek mezin e û li benda çareseriyê ye. Di hin zanîngehan de di bin banê Fakulteya Edebiyatê de, beşên Ziman û Edebiyata Kurdî hatine vekirin û hinek jî hêj li benda vekirinê ne. Di vî warî de jî gelek astengî hene û hewce ye werin çareserkirin. Ji bo çand û edebiyata kurdî beş hatine vekirin lê mixabin em dikarin bibêjin di warê cih, pirtûkxane, salonên xebat û konferansan, materyalên perwerdehiyê û hwd. de gelek kêmasî û pêdivî hene, hewce ye ev bên dîtin û bi lez bêne çareserkirin. Em di vê gotara xwe de dixwazin li ser van pirsgirêkan bisekinin û ji bo çareseriya wan hinek pêşniyazan bînin zimên. Heke ev pirsgirêkên ku di warê çand û zimên de hene, werin çareserkirin, em di vê baweriyê de ne ku; ji bo pêvajoya aştî û biratiyê wê îmkanên zor baş derkevin holê û bibin sedema biratî û aştiyê ji bo gelan.

Peyvên Sereke: Enstîtu, Edebiyat, Beş, Kurdî, Ziman, Zindî

\section{DESTPÊK}

Pirsgirêka Kurdî, em dikarin bibêjin di sedsala XIX. hetta berya vê sedsalê destpêkiriye. Dema ku Komara Tirkiyê di sala 1923'an de hat damezrandin bi awayekî zelal pirsgirêk derketin holê. Ji ber ku komar, li ser hîmê înkar û pişaftinê hat avakirin û ji xeynî Tirkan tu gelên din nasnekirin û gotin ji xeynî Tirkan li ser vê axa pîroz hindikayiyên nemisilman hene û ji Kurd, Ereb, Çerkez û hwd. netewan re gotin hûn hemû Tirk in. Bi salan ev fikr û ramana nerast pejirandin û ji Kurdan re gotin: "Hûn Tirkên çiyayî ne, dema hûn li ser berfê geryane lingên we dengê kart-kurt derxistî ye ji bo vê sedemê ji we re gotine Kurd". Li ser deşt û çiyayên Kurdan "Ne mutlu Türk'üm diyene / Ê bêje ez Tirk im kêf kêfa wî/ê ye." dinivîsandin û her roj zarokên kurdan hînî derewan dikirin û bi zarokan ev gotina "Türküm, doğruyum, çalışkanım...- Tirk im, durust im, xebatkar im..." nerast didan gotin. Zarokên kurdan jî digotin: "Em Tirk nînin gelo çima van gotinan bi me didin gotin?".

Kurdan jî, li dijî van fikrên nebaş reaksiyon çêdikirin û nerazîbûna xwe nişan didan. Ev yeka hanê jî dibû sedema şikence û muamela nebaş û bi sedan kurd diketin hepsan û zîndanan. Ev fikra pişaftin û nijadperestiyê, di vî welatê gelek xweş û şîrîn de ewletî nehîşt û bu sedemê qetlîaman û bi hezaran kes hatin kuştin, birîndarkirin, zarok bê dê û bav man, bi hezaran gund hatin şewitandin, bi mîlyonan pere hatin xerckirin û di warê aboriyê de gelek qelsî derketin holê. Lê rêvebirên Komarê dîsan jî nikarîbûn vê pirsgirêkê çareser bikin û çareser nekirin jî. Her carê bi eynî rê û rêbazan dixwestin van pirsgirêkên dîrokî çareser bikin, lê dawî li pirsgirêkan nedihat û her diçû ev pirsgirêk zêdetir dibûn. Jê re çareyek nû lazim bû, gelo çareyek çawa? Rêvebirên komarê gelek caran ev pirsa hanê ji xwe dikirin û em di vê baweriyê de ne ku wan bersiva wê dizanîbû lê nedigotin. 
Gelek caran rewşenbîrên Kurdan û Tirkan rê nîşanî rêvebirê komarê didan, lê mixabin ew her carê bi xayîntiyê dihatin tewanbarkirin û cihên wan dibû heps û zindan. Hinekan nedixwestin ev pirsgirêkên ku zirarê didin him kurdan him jî tirkan werin çareserkirin. Hinek kes dibêjin, gelek destên tarî yên bi qilêr û gemar naxwazin ev pirsgirêk werin çareserkirin, ji bo ku ew ji vê kaniya bi gemar û qirêj avê vedixwin, çekan difroşin û pê dewlemend dibin. Lê dîsa jî rewşenbîran dev ji çareseriyê bernedan û rêya çareseriyê li her der û zemanî bi qêrîn û zarîn gotin.

Gelek siyasetmedar jî di wê baweriyê de bûn ku, ev pirsgirêka kurdan bi çekan nayê çareserkirin. Mixabin gelek ji wan bêcesaret bûn û nedixwestin serê xwe ji bo vê pirsgirêkê têxin belayan û dixwestin di derbarê vê mijarê de eqlê selîm ji wan dûr bikeve û serê xwe neêşînîn. Ji wana hinekan cesareta xwe top dikirin û digotin em ê vê pirsgirêkê çareser bikin, lê mixabin wan jî mîna marşa mehterê ya Împaratoriya Osmaniyan du gav bi pêşve diavêtin gavek jî şûnde. Bo nimûne, serokwezîrê berê Silêman Demîrel dema ku hukumeta xwe ava kir çû Diyarbekrê û got: "Ez wek serokwezîr realîteya we kurdan nas dikim." lê li paş vê gotina xwe nesekinî û ji xeynî vê gotinê tu karek din nekir, serokwezîrê kevn Mesut Yilmaz jî hat Amedê û wiha axivî: "Rêya Ewrûpayê di Amedê re derbas dibe." Tansu Çiller jî got: "Em dikarin ji bo çareseriya pirgirêka kurd Modela Baskê ji xwe re bingeh bigrin $\hat{u}$ li ser qisebikin." Mixabin di dawiya axaftina wan de pirsgirêk çareser nebû û zêdetir li hev ket û carek din şer, pevçûn, qetlîam çêbûn, zarok man sêwî, gund hatin şewitandin û qetlên faîlên meçhulî zêdetir bûn.

Serokê kevn Erbaqan got: "Eger hûn bibêjin 'çi şanaziyeke bo kesê ku dibêje ez Tirk im' ew ê kurd jî bibêjin ku 'çi şanaziyeke bo kesê ku dibêje ez Kurd im' " Ev gotina Erbaqan bû sedemek ji sedemên girtina Partiya Refahê.

Serokkomarê berê, Rêzdar Turgut Ozal jî xwest vê pirsgirêkê çareser bike lê mixabin li ser wî jî gelek dek û dolab hatin çêkirin û di dawiyê de ew jî bi qilo pilo koçî dawî bû û çû.

\section{REWŞA ZIMANÊ KURDÎ}

Komara Tirkiyê ji sala 1992'an vir de di warê pirsgirêka kurdî de rastî pirsgirêkek nû hat. Ev pirsgirêk jî ev e ku; kurd dixwazin di xizmetên gelemperî de zimanê xwe bi kar bînin. Yanî kurd dixwazin di meclîsê de, di perwerdehiyê de, di çapemeniyê de, di rêvebiriyên herêmî de, di dadgehan de, di mizgeftan de, di warê hunerê de û hwd. zimanê dayîka xwe bi kar bînin. Ev daxwaza hanê jî dide xuyakirin ku, pirsgirêk ketiye merhaleyek nû.

Komara Tirkiyê, bi taybetî ji sala 2002'an û vir de hukûmetên Partiya AKPê dest bi pêvajoyek nû kir û xwest ku zimanê kurdî di çapemeniyê de, di perwerdehiyê de, di hunerê de û di siyaset û propogandayê de were bi kar anîn lê sînorê vî mafê hanê jî diyar kir û got bira bi tenê di warê çandê de were bikar anîn. Ji bo vê sedemê rêvebirên komarê bi çavên mafê takekesî û çandî di çarçoveya folklorî de li zimanê kurdî mêze kirin.

Çawa ku me li jorê jî got, daxwaza kurdan ne bi tenê bi kar anîna ziman di çarçoveya takekesî de ye. Ew dixwazin zimanê xwe, di hemû aliyên jiyanê de bixebitînin. Rêvebirên komarê pirsgirêka zimanê kurdî weke tiştek teknîkî didîtin û digotin eger em di warê teknîkî de hinek astengan ji holê rakin, ev pirsgirêk dê were çareserkirin. Lê kurd jî wisa nafikirin, dibêjin hewceye mirov li vê pirsgirêkê bi çavê mafê mirovan mêze bike û bi wî azînê 
tevbigere û dibêjin hewceye zimanê kurdî ji sinorê çandê derbas bibe û di warê siyasî de cihê xwe bi awayek xurt bigre.

Dewlet, bi çavê teknîkî li vê pirsgirêkê mêze dike û kurd jî bi çavê mafê mirovatiyê lê mêze dikin. Bi çi çavî lê tê mêzekirin, bila were mêzekirin, em îro dibînin ku zimanê kurdî hêdî hêdî di warê xizmeta gelemperî de cihê xwe digre.

Di dîroka Komara Tirkiyê de Zimanê Kurdî cara yekem di sala 1991'î de, li parlamentoya Enqerê ji devê parlementera partiya DEP'ê ya Diyarbekirê Rêzdar Leyla Zana ve dema sond xwarina bo parlamenteriyê de, bi wê hevoka ku di dawiya sondxwarina xwe de bi kurdî gotibû: "Ez vê sondê ji bo Gele Kurd ĥ Tirk dixwûm." û piştre ket rojeva siysetê. Dema Zana, ew hevok bilêv kir kevir û kuçik lê hatin barîn û ew û hevalên wê bi salan di heps û zîndanan de man û di sala 1994'an de namzetiya wan ji destên wan girtin û partiya wan jî dadan. Piştî ku 19 sal di ser vê bûyera nebaş re derbas bû, di warê zimên de du bûyer qewimîn: Ya yekemîn ew bû ku; di meha Adara 2010'an de, parlementerê partiya BDP'ê di derbarê mafê nivîsandina zimanê kurdî de pêşniyazek da parlementoyê. Dema ev pêşniyaz di parlementoyê de hat xwendin qedexekirina li ser Zimanê Kurdî hate qulkirin û navê Şivan Perwerî bi tîpa "w" yê hate nivîsandin. Hetanî wê demê bikaranîna tîpên "q, w, x" qedexe bûn û bikaranîna wan dibûn sedemê ceza dayînê. Hetanî wê gavê eger hevokek kurdî di meclîsê de bihata gotin di zabitnameyan de weke "zimaneke ku nayê zanîn" dihat qeydkirin. Ez nizanim gelo bi zanîn an bi nezanî, bi awayê rasteqînî, yanî di kurdî de çawa derbas dibe wisa jî di zabitnameyan de hate qeydkirin. Ev rewşeke girîng û merhaleyek nû bû.

\section{Dİ QADA PERWERDEHIYŶ DE REWŞA ZIMANÊ KURDÎ}

Ji bo ku mijar baş derkeve holê û bi awayek zelal were fêmkirin em dixwazin vê mijarê di bin sê bendan de binirxînin:

\subsection{Ji Bo Perwerdehiya Zimanê Kurdî Qursên Taybet}

Cara yekem li Tirkiyê bi awayekî qanûnî destûra qurswên taybet ji bo Zimanê Kurdî hate dayîn. Di 20'ê Tebaxa 2002'an de, di rêziknameya bi navê "Perwerdehiya ziman $\hat{u}$ zaravayên cûda yên ku hemwelatiyên Tirkiyeyê di jiyana xwe ya rojane de bi awayekî kevneşopî de dixebitînin." de guhertin hate çêkirin û vekirina qursên taybet, ji bo Zimanê Kurdî hêsan bû. Em di wî baweriyê dene ku ev guhertina hanê di derbarê krîterên Yekîtiya Ewrûpayê de hate çêkirin. Lê di gel van guhertinan dîsan jî rêvebirên karên perwerdehiyê astengên cuda cuda derxistin. Ew hincetên rêvebirên perwerdehiyê jî hatin çareserkirin û di meha Nîsana 2004'an de li bajarê Wan'ê, qursa Zimanê Kurdî dest bi perwerdehiyê kir. Kapasîteya vê qursê bi qasî girtina 480 xwendekarî hebû. Mixabin 82 kesî serî li qursê dan, li Batmanê jî di meha Nîsana 2004'an de bi beşdarbûna nêzîkî 30 hezar kesî qursa Zimanê Kurdî hat vekirin. Paşê jî li pê hev li bajarên cur be cur qurs hatin vekirin, mixabin ji ber hin bêderfetiyan qursên vekirî jî yek bi yek hatin girtin.

Ev bûyer danî xwuyakirin ku; eger dewlet bi awayekî fermî, alîkariya van qursan neke tu serkeftin çênabe. Ji ber ku gelê herêmê, di warê aboriyê de di rewşek nebaş de ye û ew nikarin bihayê qursê jî bidin. Ya duyem jî; dema ku wan ev qurs xelas kirin û belgeyên xwe wergirtin, ev belge bo kar û xebatekê wê bi kêrî wan neyê. Hewceye ku di jiyana rojane de ji bo van belgeyan wateyek hebe û bi kêrî tiştekî were. 
Ji aliyek din ve jî gel difikirî û digot: 'gelo em ê çima zimanê dayika xwe bi peran û di qursên taybet de fêr bibin?'. Ev rewşa hanê ji xwe re weke heqaret didîtin û li dijî vê bertek nîşan didan. Kurdan dev ji van qursên taybet berdan û fikra perwerdehiya bi zimanê dayikê anîn ziman û gelek çalakî li dar xistin. Kurdan gotin ku; gerek zarokên me di dibistanan de bi zimanê xwe perwerde bibin!

Kurdan digot; eger dewlet alîkariya vî karê hanê neke zimanê kurdî di bin xeterek mezin de ye û rewş wiha berdewam bike wê Zimanê Kurdî mîna hinek zimanan bibe zimaneke mirî. Zimanê Kurdî, bi qursên taybet nikare li ber xwe bide, li ser lingan bisekine û zindîbûna xwe bidomîne. Eger ziman di jiyana gelêrî de tune be, bi teqez temenê wî kêm e.

\subsection{Mafề Perwerdehiya Zimanê Zikmakî}

Kurd, di Komara Tirkiyê de xwe ne wekî hindikayî weke unsurên eslî dibînin, ji bo vê sedemê di warê zimên de jî, bi vî awayî difikirin û dibêjin ku: "Mafề perwerdehiya bi zimanê zikmakî mafê me ye jî̀'. Em vê daxwaza hanê ya di rê de di serê salên 2000'an de zêdetir dibînin. Di wan salan de, xortên kurdan ên ku di zanîngehan de dixwendin serî li serokatiyên beșên ku têde dixwendin didan û digotin; em dixwazin bi zimanê dayîka xwe perwerdehiyê bibînin. Sed car heyf ew xortên ku serî li serokatiyên beşên xwe didan, yan têkiliyên wan ji zanîngehên wan dihate qutkirin yan jî, bi salan ceza diwxarin û bersiva daxwaza wan dibûn heps û zindan. Lê gelê Kurd dest ji daxwaza xwe bernedan û di destpêka perwerdehiya sala 2010-2011'an de li gelek deverên ku kurd lê hebûn, di hefteya yekemînde çalekiyek li dar xistin û navê wê jî danîn; "serkêşiya sivîlî" yan jî "boykotkirina dibistanan" û zarokên xwe neşandin dibistanan.

Hinek kes li dijî vê xwestekê derdiketin û bihaneyên wan jî bi gelemperî ev bûn: Ne mumkun e ku zimanê kurdî bibe zimanê perwerdehiyê, ji ber ku zimanê kurdî ji bo zanistiyê ne dewlemend e, gel perçe perçe dike, dibe sedem ku kurd û tirk ji hev û din fêm nekin, yekîtiya komarê xerab dike, ne mumkune ku ji bo perwerdehiya zimanê kurdî binesazî were amadekirin û ji bo vî karî jî mesrefek giran lazim e, zarokên ku bi kurdî perwerdehîyê dibînin di jiyana gelemperî de nikarin kar bikin, eger ev daxwaz were cih dibe sedemê zimanê fermî yê duyemîn di jiyana gelemperî de û hwd. em dikarin van bihaneyan zêde zêde dirêj bikin lê ne hewceye.

Li vir du rayên sereke derdikevin holê, ji wana yek perwerdehiya bi zimanê zikmakî; ya duduyan jî, perwerdehiya bi yek zimanî yanê bi zimanê Tirkî. Kurd dibêjin, eger Zimanê Kurdî nebe zimanê perwerdehiyê; di pêşerojê de ewê di bin bandora zimanê Tirkî de bimîne û di nav çend salan de wenda bibe. Zimanê Kurdî ruhê kurdayetiyê ye, rûmeta kurdan e, eger ziman biçe wenda bibe wê kurd jî wenda bibin. Eger kurd dixwazin neyên pişaftin û weke miletek ji miletên dinyayê jiyana xwe berdewam bikin, hewceye li zimanê xwe xwedî derkevin û perwerdehiyê bi zimanê dayîka xwe bibinin.

Raya duduyan jî dibêjin, eger em mafê perwerdehiya bi zimanê zikmakî bidin kurdan; ev biryar dibe sedemê perçebûna welatê me, kurd û tirk ji hev cuda dibin û nikarin têkiliyan bi hev re deynin û ji hev û din fêm bikin.

Em di vê baweriyê de ne ku, ramana yekemîn rast e û ya duyemîn jî çewt e. Eger mafê zimanê zikmakî were dayîn ev biryara hanê wê bibe sedemê desthevgirtina kurdan û tirkan. 
Ev daxwaza hanê daxwazek însanî ye û di rêde ye. Bi sedsalan e kurd bi vî zimanî qisedikin û jiyana xwe didomînin. Ew ê çima bibe sedemê perçebûna welat! Zarokên kurdan hetanî heft saliya xwe bi kurdî diaxivin û dema diçine dibistana seretayî mêze dikin ku mamosteyê/a wan bi zimanekî cuda qise dike û ew ji tu gotinên wî/wê fêm nakin. Ev jî dibe sedemê tengasiyek gelek mezin ji bo zarokan û psîkolojiya wan zêde zêde xerab dike.

Fikra duyemîn çewt e, ji bo ku ew kesên vî fikr û ramanî derdixînin pêş, fikarek wiha tê hişê wan: Eger ev maf were dayîn wê welatê me perçe perçe bibe! Em dikarin bibêjin ev fikara hanê fikarek ne di rê de ye. Ev nabe sedemê perçebûyînê, berevajî eger ev maf neyê dayîn ew ê bibe sedemê perçebûyinê. Ji bo ku dema ev mafề însanî û mirovahî were dayîn wê kurd bifikirin û bibêjin ev komara hanê ya me ye jî çi maf daye gelê tirk daye me jî, hewceye ku em jî bi çavek baş li komarê mêze bikin û lê xwedî derkevin.

\subsubsection{Di Zimanê Kurdî De Çapemenî}

Kurdan bi zimanê xwe, berya avakirina Komara Tirkiyê gelek berhem nivîsandine. Piştî avakirina komarê zordariyek mezin li ser çapemeniya kurdî çêbûye û em dikarin bibêjin hetanî 1948'an tu berhemek bi kurdî û bi tîpên latînî nehatiye nivîsandin. Di sala 1959'an de dema Musa Anter ji sedema doza 49. di girtîgehê de bû, metnek şanoyê bi navê "Birîna Reş" nivîsî û di sala 1965'an de ev berhem da weşandin û pişt re jî, bi dizî hinek berhem hatin çapkirin. Dema Kenan Ewren di 12'ê Îlona 1980'yî de înqilab çêkir, wekî hemû aliyan li ser çapemeniya Kurdî jî zor û zext zêdetir bûn lê li derveyî welêt kurdên rewşenbîr gelek pirtûk çapkirin û belav kirin. Di piş van tiştan re televîzyonên kurdî dest bi weşanan kirin, di sala 1995 'an de Med TV dest bi weşanê kir û paşê jî nêzîkî 15 TV'an dest bi weşanên xwe kirin.

Di 2'yê Berfanbara 2009'an de di bin TRT'ê de qanalek kurdî bi navê TRT 6'ê bi zaravayê Kurmancî û Zazakî dest bi weşanê kir. Li hember vê biryarê hinek kesan bertekên erênî, hinekan jî bertekên neyînî nîşan dan. Yên ku bertekên erênî nîşan didan digotin ku, ev gava hanê gavek demokratîk e; yên bertekên neyînî nîşan didan jî digotin, ev fikra hanê rengek ji rengê cerdevaniya çandî û siyasî ye.

Dema TRT 6'ê dest bi weşana xwe kir pirsgirêkek kevnar e jî bi wê re derket holê; rêvebirên dewletê di derbarê elfabeya kurdî de digotin ku tîpa "q, x, w" di elfabeya tirkî de tune ye ji bo vê sedemê hewceye ev tîp neyên bikar anîn, eger were bikar anîn wê bibe sedemê perçebûna welêt! Bi TRT 6'ê re azadî ji van tîpan re hat û bi awayek zelal ev tîpan hatin bikaranîn. Bo nimûne navê Van'ê weke Wan, navê cejna nevrûzê weke Newroz, navê Karsê weke Qers û navê Hude weke Xwedê hate bikar anîn.

\section{Di Zanîgehan de Perwerdehiya Bi Zimanê Kurdî}

Weke me li jorê jî gotibû; Kurd dixwazin ku di hemû aliyên xizmeta gelemperî de Zimanê Kurdî were bikar anîn. Keç û xortên kurdan ji bo bi zimanê dayika xwe perwerdê bibînin daxwazname dan serokên beşên xwe, lê mixabin bersiva daxwaza wan yan heps û zîndan, yan jî qutkirina têkiliyên wan ji zankoyên wan bûn.

Di Mijdara 2008'an de Serokê Lijneya Hindekariya Bilind (YÖK) Rêzdar Yûsuf Ziya Özcan got : "Eger ji bo avakirina beşa Ziman û Wêjeya Kurdî daxwaz werin kirin em ê van 
daxwazan binirxînin". Rêvebirên YÖK'ê digotin ku di warê qanûnê de li pêşiya vê xwestekê tu asteng tune ne û daxwazkirina zanîngehan ji bo vekirina van beşan ji bo me bes e. Li ser van gotinan Rektorê Zanîngeha Mardin Artûklûyê Rêzdar Prof. Dr. Serdar Bedîi Omay, daxwuyaniyek da çapemeniyê û got di derbareyê vê mijarê de me hinek tişt amade kirine û ji bo vekirina beşê emê di demeke kin de serî li YÖK'ê bidin. Hinek zanîngehên din jî weke Rektorê Zanîngeha Enqerê, Şirnexê û Hekariyê jî bi nêrînek baş li vê daxwazê mêzekirin. Sed car heyf û mixabin piştî salekî YÖK'ê bersiv da û got; em naxwazin bi navê Beşa Ziman û Wêjeya Kurdî beşekê vekin lê em dixwazin bi navê "Yaşayan Diller Enstîtüsü- Enstîtûyen Zimanên Zindî’' beşekê vekin. Li gorî vê biryarê di bin navê vê enstîtûyê de li ser Zimanê Kurdî, Farisî, Erebî û Sûryanî xebat tên kirin. Rektorê Zanîngeha Mardin Artûklûyê li dijî vê biryarê bertek nîşan da û got; eger ji bo beşa zimanê Kurdî di bin fakulteyan de beşa lîsansê neyê vekirin ev kêmasiyek gelek mezin e û gotinên xwe wiha domand : "Kurdolojî zanistiyek e kevnare ye $\hat{u}$ diroka wê digêhêje 1787'an $\hat{u}$ hetanî vê gavê di 30 zanîngeh, enstîtû, beş, navend, kursî $\hat{u} h w d$. de tê fêrkirin. Lê mixabin di welatê me de bi navê xwe nayê avakirin, ev jî nakokiyek mezin e $\hat{u}$ şirovekirina vê jî gelek zor e. Eger ku ev beş ne bi navê Kurdolojiyê bi navê 'Enstîtûyên Zimanên Zindî' were binavkirin, ev dibe ku bibe sedema dilşikestiniya hemwelatiyên me yên Kurd."

Dîsa di Îlona 2009'an de Rektorê Zanîngeha Stenbolê Rêzdar Prof. Dr. Yusuf Söylet jî di derbarê "pêvajoya vebûnê de" got; me biryar da ku em di zanîngeha xwe de beşa Kurdolojiyê vekin. Lê serokê YÖK'ê Birêz Özcan got ku pêşikiya me bi zimanê Kurdî nîne bi zimanên din hene. Li gorî wî hewceye berî vekirina vê beşê beşa Zimanê Tirkî, Farisî û Erebî were vekirin û were xurtkirin. Ji xwe li gorî Birêz Özcan Zimanê Kurdî ji \% 60-70 Farisî ye û ji \% 20-25 Erebî ye û hinek peyv jî ji Zimanê Tirkî hatine girtin. Bi rastî Birêz Özcan, bi zimanek akademik digot Zimanê Kurdî ne zimanek bi serê xwe ye, ji bo vê sedemê bi awayek sergirtî digot nehewceye Kurdolojî were vekirin.

Di 10'ê Berfanbara 2009'an de li Mêrdînê di Zanîngeha Artukluyê de cara yekemin bi awayekî fermî navê wê Kurdolojî nebe jî, bi navê "Enstîtuya Zimanên Zindî" enstîtuyek hate avakirin û di vê enstîtuyê de Beşa Ziman û Çanda Kurdî, Beşa Ziman û Çanda Suryanî û Beşa Ziman û Çanda Erebî hate sazkirin. Paşê jî weke, li Dêrsim, Bîngol, Muş, Sêêrt û Wan'ê ev enstîtu hatin vekirin. Em dikarin bibêjin zimanê Kurdî di xizmeta gelemperî de bi awayek berfireh cih girt.

\section{ENCAM}

Me di vê gotara xwe de xwest ku em berê, li Tirkiyê hinekî behsa rewşa Zimanê Kurdî bikin û paşê jî ji bo çareserkirina pirsgirêka kurdî û pêvajoya aşîtiyê hinek pêşnîyazan bînin ziman. Weke tê zanîn pirsgirêka kurdî pirsgirêkek kevnare ye û hewceye bi lez û bez were çareserkirin. Di çareserkirina vê pirsgirêkê de girîngiya herî mezîn ziman e. Eger ev pirsgirêk were çareserkirin gelek aliyên pirsgirêkê çareser dibin û pêvajoya aşitiyê bi awayekî serkeftî bi dawî dibe. Li gorî hizr, fikr û ramanên me, ev tiştên ku em li jêr dibêjin eger werin çareserkirin ew ê bi awayek baş barê pêvajoyê sivik bibe. Divê ev karên li jêr tene gotin ger werne bi cih anîn wê ew baweriya gel carek din xwurt bibe. 
1. Ew astengiyên ku li pêşiya perwerdehiya zimanê kurdî hene bi awayek baş bên paqij kirin û him di mêjûyê rêvebirên komarê de zelaliyek bê çêkirin him jî dilê kurdan yê şikestî were xweşkirin. Nehewceye ku rêvebirên komarê zimanê kurdî ji xwe re weke hevrik bibîne û cudahiyê têxine navbera zimanê kurdî û tirkî. Divê zimanê kurdî piçûk neye dîtin û weke tê gotin "ev ne zimanek serbixwe ye û tevlihevkirina hinek zimanan e, ne zimanê şarezayî û zanistiyê ye, zimanê çiyayiyan" e lê heqaret neyêne kirin û hewceye firsendê bide zîmanê kurdî ku ew di jiyana gelemperî de bi hemû alîyan ve cihê xwe bigre. Weke tê zanîn zimanê kurdî van gotinan heq nake û ew jî weke zimanên dinyayê zimanê gelekî ye û bi vî zimanî di warê edebiyat, felsefe û hunerê de gelek berhem hatine nivîsandin û bi mîlyonan kes bi vî zimanî diaxivin.

2. Ew kesên ku di van sazgehan de dixebitin, hewceye rêvebirên komarê bi her aliyî de bi wan bawer bin û di derbarê niyeta wan de tiştên weke; gelo armanca van kesan çî ye, gelo dubendiyê dixwazin têxin nava gel, dixwazin welêt perçe bikin û hwd. nefikirin û wan kesan rencîde nekin. Ji bo ku ew kesên ku di van sazgehan de dixebitin ew jî weke akademîsyen û karmendên sazgehên din karên xwe dikin. Yanê gerek xebatkar dema karên xwe dikin bila fikar nekin û nebêjin gelo rêvebirên zanîngeha me di derbarê me de çi dibêjin û çi difikirin. Bi kurtasî hewceye ewletî û bawerî di navbera herdu aliyan de jî hebe.

3. Hewceye navê Enstîtuya Zimanên Zindî were guhertin û yan bibe Enstîtuya Kurdolojiyê yan jî bibe Enstîtuya Ziman û Edebiyata Kurdî. Çawa ku dema di TRT yê de qanalek hat vekirin nav lê hat danîn TRT 6 û paşê jî hate guhertin û navê wî bû TRT Kurdî, ev guhertina hanê zêde zêde di rê de bû wisa jî navê enstîtuyan bên guhertin. Ji bo ku dile kurdan hinek nerm û germ bibe.

4. Ji bo van sazgehan gerek avahiyên serbixwe bên çêkirin. Hewceye ev enstîtu jî mîna enstîtuyên din di bin banê xwe de bin. Eger ev wiha be enstîtu wê xwe bi qedr û qîmet bibînin û bi moralek xweş û geş karên xwe bidomînin. Ger odeyên mamosteyan berfireh û cuda nebin, karmendên sazgehê hemû li ser hev, di odeyek du odeyan de bicivin û kar bikin ev tiştek gelek nexweş û xirab e. Weke beşên din polên dersdayînê bila aîdî beşê bin û hewcedarî bi çend polan hebe, ewqas pol bên vekirin.

5. Pirsgirêka qadroyan hewceye bi awayek lez û bez were çareserkirin. Eger di sazgehek de bi qasî hewcedariyê mamoste tunebin, baweriya xwendekaran bi wê beşê nayê û ew beş bi awayek rêk û pêk nikare bi ser bikeve. Ji bo ku van beşan cara yekem li Tirkiyê de dest bi xizmetê kirine hewceye bi çavek pozîtîf li wan were mêzekirin û wekî beşên din zêdetîr qadro were dayîn ji bo ku bikaribin li ser lingên xwe bisekinin.

6. Ji bo Kurdolojiyê pêwiste ku bi awayek zû di hemû zanîngehên ku tê de ev beş heye pirtûkxane werin avakirin. Ji bo avakirina pirtûkxaneyan hewceye zanîngeh di aliyê aboriyê de alîkariya rêvebirên enstîtuyan û mamosteyên wan bikin û butceyek taybet jî were veqetandin, hewceye enstîtu jî di vî aliyî de alîkariya hevûdin bikin û çavkaniyên li cem xwe bi hevûdin re parve bikin. Hewceye qomîsyon bên avakirin û ji bo çavkaniyên ku li derveyî welêt in û li welatên weke Rûsya, Ermenîstan û Başûrê Kurdistanê ne bên anîn. 
7. Di navbera enstîtuyan de têkilî werin xurtkirin, çûn û hatin were çêkirin, ji bo ku kî çi dike ji aliyê yên din ve were zanîn.

8. Hewceye ders bi kurdî werin dayîn, xwendekar jî di dawiya perwerdehiyê de bi awayekî zorbaş hînî Zimanê Kurdî bibin.

9. Ji bo ku xwendekarên jîrek werne van beşan hewceye bi awayek garantî, soz were dayîn ku dema wan, ev beș qedandin bêkar nemînin. Di serî de gotin ên ku van beşan diqedîne em wan dikin mamosteyên Zimanê Kurdî lê mixabin rêvebir li soza xwe xwedî derneketin. Nêzîka du-sê hezar xwendekar van beşana qedandine lê hetanî vê gavê 20-30 kes weke mamoste hatine tayînkirin. Eva jî dibe sedemê bêbawerîtiyê.

10. Hewceye di dibistanan de bi awayekî bêtirs hemwelatiyên kurd bikaribin di nava dersên hilbijarte de dersa Zimanê Kurdî hilbijêrin. Dema ev ders hilbijartin, bira fikar nekin û nebêjin rêvebirên dibistanê gelo ji zarokên me re tiştek nebaş dibêjin yan na. Lazime ku hemwelatî û xwendekar bi awayek kêfxweşî bikaribin van dersan hilbijêrin û bixwînin.

11. Di zanîngehan de dema xwendekaran xwestin ji dersên hilbijarte dersa Zimanê Kurdî hilbijêrin, hewceye hem rêvebirên wan fakulteyan him jî rêvebirên enstîtuyan li peşiya wan astengan dernexînin û ji wan re bibin alîkar û karên wan hêsantir bikin.

12. Hewceye siyasetmedar li ser vê pirsgirêkê bisekinin û ji bo zimanê Kurdî bibe zimanê perwerdehiyê xebatan bikin û ji îro de ji bo vî karî xwe û gel û sazgehan amade bikin.

13. Ya herî girîng ev e ku hewceye ev kar bi lez û bez werin kirin. Ji bo ku baweriya gel ji hêla pêvajoya aştiyê ve roj bi roj diçe kêm dibe û aramiya wan dibe ku xelas bibe û baweriya wan qet nemîne.

Hewceye evan kêmasiyan li demê neyê belavkirin û hindik hindik neyên dayîn û di carekî de were dayîn. Ji bo ku bibe sedem qîmet û bibe xwedan rûmet. 